ISSN: 2638-5279

Volume 3, Issue 2, 2020, PP: 01-03

\title{
Using GH-Method: Math-Physical Medicine to Conduct Segmentation Analysis to Investigate the Impact of Weight on Fasting Plasma Glucose (No: 66)
}

\author{
Gerald C. Hsu \\ eclaireMD Foundation, USA.
}

*Corresponding Author: Gerald C. Hsu, eclaireMD Foundation, USA.

\section{INTRODUCTION}

The dataset is provided by the author, who uses his own type 2 diabetes (T2D) metabolic conditions control, as a case study via the "math-physical medicine" approach of a non-traditional methodology in medical research.

Math-physical medicine starts with the observation of the human body's physical phenomena (not biological or chemical characteristics), collecting elements of the disease related data (preferring big data), utilizing applicable engineering modeling techniques, developing appropriate mathematical equations (not just statistical analysis), and finally predicting the direction of the development and control mechanism of the disease.

This paper is based on a big data collected from a period of 1,418 days (from $6 / 1 / 2015$ to $4 / 19 / 2019$ ) with 2,836 data of weight and FPG. This dataset is provided by a long-term type- 2 diabetes patient under a lifestyle management program.

\section{METHOD}

Initially, the author conducted a time series analysis between weight and FPG, which he found a high correlation $(\mathrm{R}=65 \%)$ existing between these two curves. Then, he used a trial-and-error method to find two suitable dividing lines of weight at $170 \mathrm{lbs}$. (BMI 25) and FPG at $107 \mathrm{mg} / \mathrm{dL}$ (under pre-diabetes). Using these two dividing lines, he further separate the entire dataset into two categories:

Low Category: Weight: 160-170 lbs. and FPG 50-107 $\mathrm{mg} / \mathrm{dL}$

High Category: Weight 170-190 lbs. and FPG 107-200 $\mathrm{mg} / \mathrm{dL}$

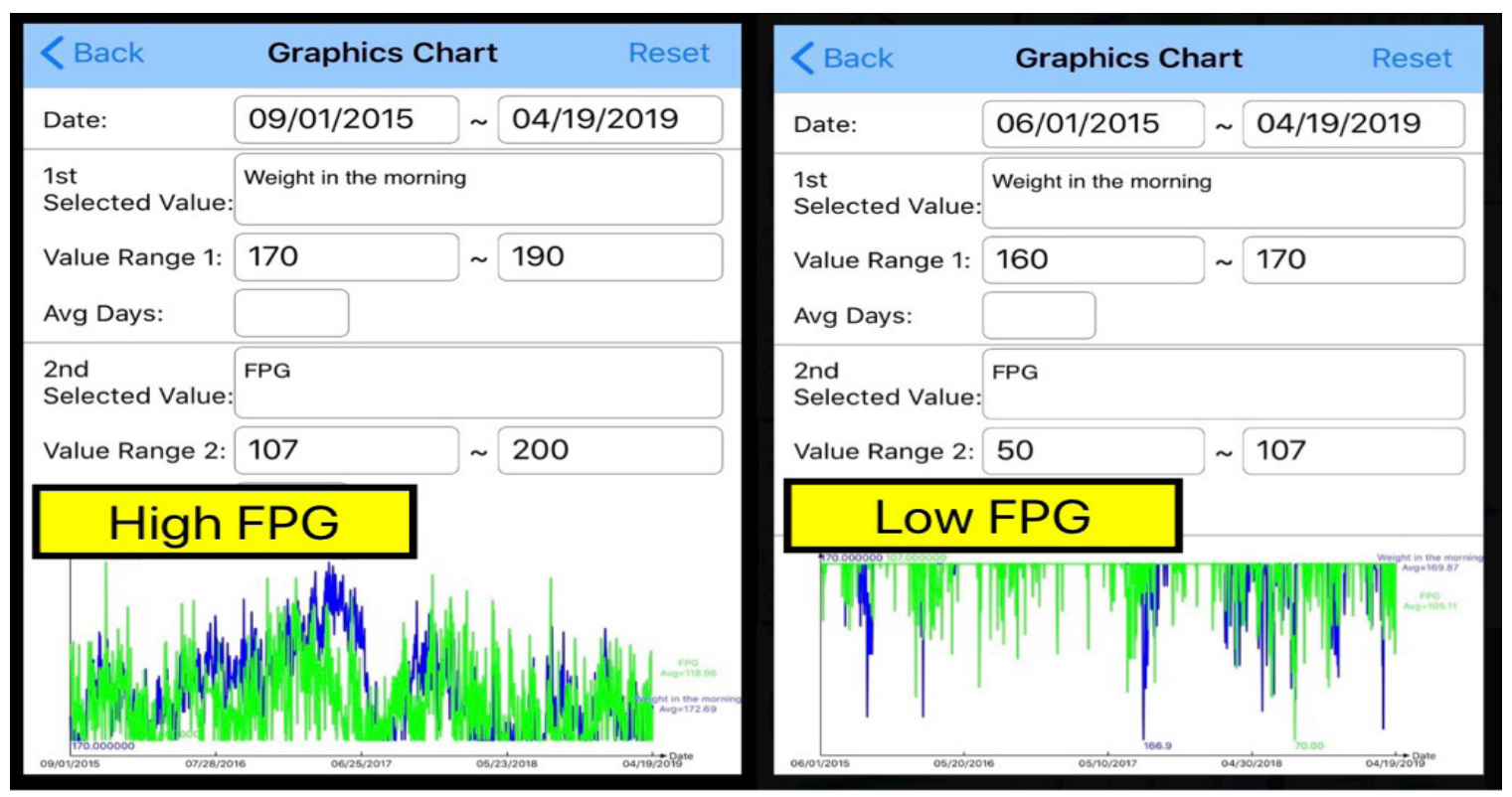

Figure1. Dividing Line and Data Range of High and Low Comparison between FPG (107 mg/dL) and Weight (170 lbs.)

Open Access Journal of Internal Medicine V3 . I2 . 2020 
Using GH-Method: Math-Physical Medicine to Conduct Segmentation Analysis to Investigate the Impact of Weight on Fasting Plasma Glucose (No: 66)

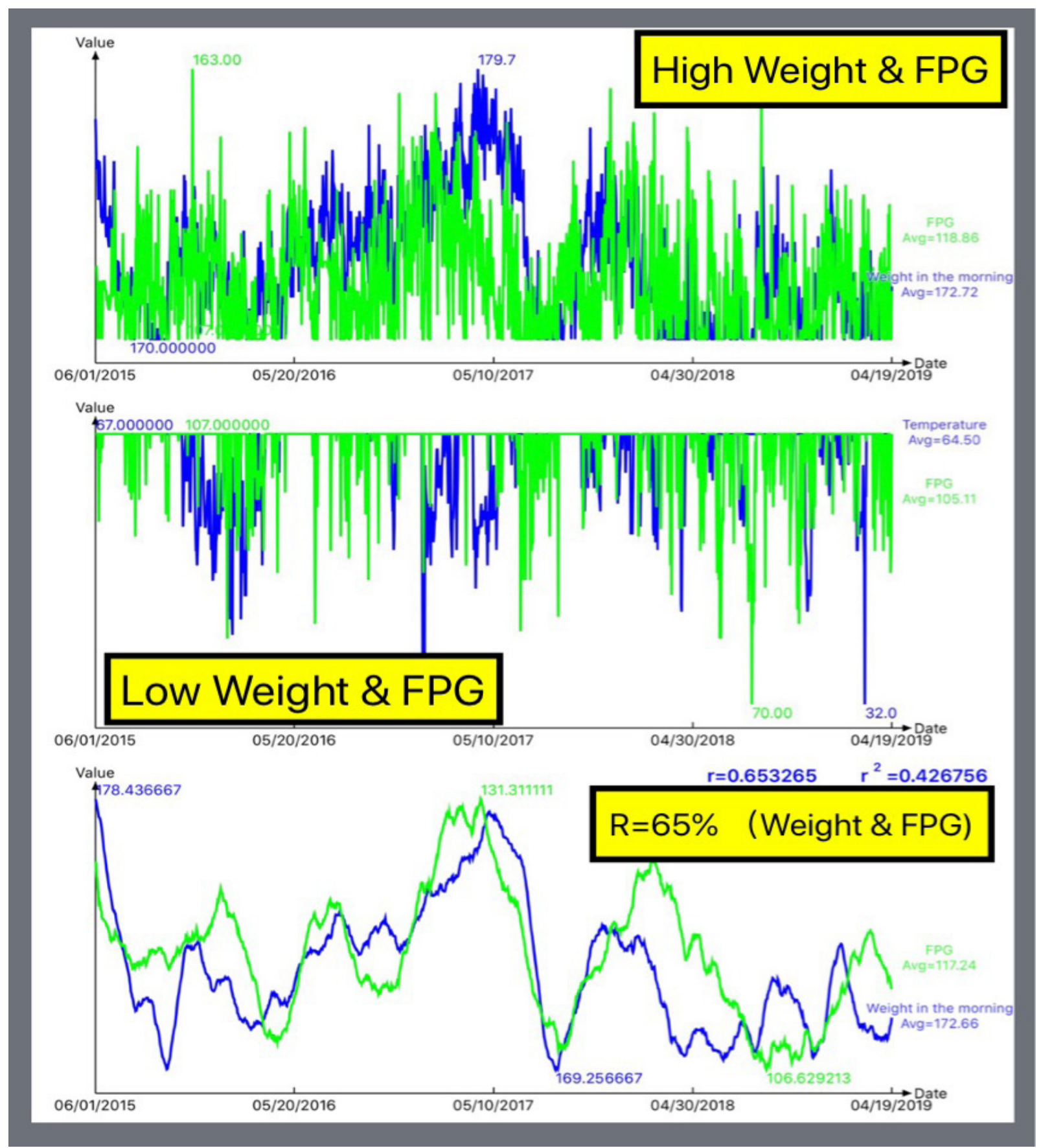

Figure2. High and Low FPG vs. Weight

\section{RESULTS}

By visual observation of these two graphs, it is obvious that weight vs. FPG are correlated with each other in both high category and low category. This was also comparable with the author's discoveries in his earlier publications. This particular T2D patient needs to keep his weight under 170 lbs. (BMI 25), so that his
FPG will be kept below $107 \mathrm{mg} / \mathrm{dL}$. This can be a very simple and useful guideline for other T2D patients.

\section{CONCLUSION}

By using the GH-Method: math-physical medicine, the author investigated the impact of FPG by segmented weight analysis. He has also developed a simple and useful guideline for other T2D patients' FPG control. 
Using GH-Method: Math-Physical Medicine to Conduct Segmentation Analysis to Investigate the Impact of Weight on Fasting Plasma Glucose (No: 66)

\section{REFERENCES}

[1] Hsu, Gerald C. (2018, June). Using Math-Physical Medicine to Analyze Metabolism and Improve Health Conditions. Video presented at the meeting of the 3rd International Conference on Endocrinology and Metabolic Syndrome 2018, Amsterdam, Netherlands.

[2] Hsu, Gerald C. (2018). Using Signal Processing Techniques to Predict PPG for T2D. International JournalofDiabetes \& Metabolic Disorders, 3(2),1-3. Retrieved from https://www.opastonline.com/ wp-content/uploads/2018/06/using-signalprocessing-techniques-to-predict-ppg-for-t $2 \mathrm{~d}$ ijdmd-18.pdf
[3] Hsu, Gerald C. (2018). Using Math-Physical Medicine and Artificial Intelligence Technology to Manage Lifestyle and Control Metabolic Conditions of T2D. International Journal of Diabetes \& Its Complications, 2(3),1-7. Retrieved from http://cmepub.com/pdfs/ using-mathphysical-medicine-and-artificialintelligence-technology-to-manage-lifestyleand-control-metabolic-conditions-of-t2d-412. pdf

Citation: Gerald C. Hsu. Using GH-Method: Math-Physical Medicine to Conduct Segmentation Analysis to Investigate the Impact of Weight on Fasting Plasma Glucose (No: 66). Open Access Journal of Internal Medicine. 2020; 3(2): 01-03.

Copyright: (C) 2020 Gerald C. Hsu. This is an open access article distributed under the Creative Commons Attribution License, which permits unrestricted use, distribution, and reproduction in any medium, provided the original work is properly cited. 